\title{
Dissecting the genome of star fruit (Averrhoa carambola L.)
}

\author{
Yannan Fan (1)', Sunil Kumar Sahu (1)', Ting Yang ${ }^{1}$, Weixue $\mathrm{Mu}^{1}$, Jinpu Wei ${ }^{1}$, Le Cheng ${ }^{2}$, Jinlong Yang ${ }^{2}$, Ranchang $\mathrm{Mu}^{3}$,
}

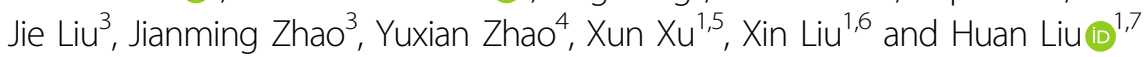

\begin{abstract}
Averrhoa carambola is commonly known as star fruit because of its peculiar shape, and its fruit is a rich source of minerals and vitamins. It is also used in traditional medicines in countries such as India, China, the Philippines, and Brazil for treating various ailments, including fever, diarrhea, vomiting, and skin disease. Here, we present the first draft genome of the Oxalidaceae family, with an assembled genome size of $470.51 \mathrm{Mb}$. In total, 24,726 protein-coding genes were identified, and 16,490 genes were annotated using various well-known databases. The phylogenomic analysis confirmed the evolutionary position of the Oxalidaceae family. Based on the gene functional annotations, we also identified enzymes that may be involved in important nutritional pathways in the star fruit genome. Overall, the data from this first sequenced genome in the Oxalidaceae family provide an essential resource for nutritional, medicinal, and cultivational studies of the economically important star-fruit plant.
\end{abstract}

\section{Introduction}

The star-fruit plant (Averrhoa carambola L.), a member of the Oxalidaceae family, is a medium-sized tree that is distinguished by its unique, attractive star-shaped fruit (Supplementary Fig. 1). A. carambola is widely distributed around the world, especially in tropical countries such as India, Malaysia, Indonesia, and the Philippines, and is considered an important species; thus, it is extensively cultivated in Southeast Asia and Malaysia ${ }^{1,2}$. It is also a popular fruit in the markets of the United States, Australia, and the South Pacific Islands ${ }^{3}$. Star fruits have a unique taste, with a slightly tart, acidic (in smaller fruits) or sweet, mild flavor (in large fruits). Star fruit is a good source of various minerals and vitamins and is rich in natural antioxidants such as vitamin $\mathrm{C}$ and gallic acid. Moreover, the presence of high amounts of fiber in these fruits aids in absorbing glucose, retarding glucose

\footnotetext{
Correspondence: Huan Liu (liuhuan@genomics.cn)

${ }^{1}$ State Key Laboratory of Agricultural Genomics, China National GeneBank, BGIShenzhen, 518120 Shenzhen, China

${ }^{2}$ BGI-Yunnan, BGl-Shenzhen, 650106 Kunming, China

Full list of author information is available at the end of the article.

These authors contributed equally: Yannan Fan, Sunil Kumar Sahu
}

diffusion into the bloodstream and controlling the blood glucose concentration.

In addition to its use as a food source, star fruit is utilized as an herb in India, Brazil, and Malaysia and is widely used in traditional Chinese medicine preparations ${ }^{4}$ as a remedy for fever, asthma, headache, and skin diseases ${ }^{5}$. Several studies have demonstrated the presence of various phytochemicals, such as saponins, flavonoids, alkaloids, and tannins, in the leaves, fruits, and roots of star-fruit plants $^{6,7}$; these compounds are known to confer antioxidant and specific healing properties. A study by Cabrini et al. ${ }^{5}$ indicated that the ethanolic extract from $A$. carambola is highly effective in minimizing the symptoms of ear swelling (edema) and cellular migration in mice. A flavonoid compound (apigenin-6-C- $\beta$-fucopyranoside) isolated from $A$. carambola leaves showed antihyperglycemic action in rats, and might show potential for use in the treatment and prevention of diabetes ${ }^{8}$. Moreover, 2-dodecyl-6-methoxycyclohexa-2,5-diene-1,4dione (DMDD) extracted from the roots of A carambola exhibits potential benefits in the treatment of obesity, insulin resistance, and memory deficits in Alzheimer's disease $^{9,10}$. 
Although $A$. carambola plays very significant roles in traditional medicine applications, there are very limited studies on $A$. carambola at the genetic level, mainly due to a lack of genome information. Therefore, filling this genomic gap will help researchers to fully explore and understand this agriculturally important plant. As a part of the 10KP project ${ }^{11,12}$, the draft genome of $A$. carambola collected from the Ruili Botanical Garden in Yunnan, China, was assembled in this study using an advanced 10X genomics technique to further elucidate the evolution of the Oxalidaceae family. A fully annotated genome of $A$. carambola will serve as a foundation for pharmaceutical applications of the species and the improvement of breeding strategies for the star-fruit plant.

\section{Results}

\section{Genome assembly and evaluation}

Based on k-mer analysis, a total of 35,655,285,391 kmers were used, with peak coverage of 75 . The $A$. carambola genome was estimated to be $\sim 475 \mathrm{Mb}$ in size (Supplementary Fig. 2). To perform genome assembly, a total of $156 \mathrm{~Gb}$ of clean reads were utilized by Supernova v2.1.1 ${ }^{13}$. The final assembly contained 69,402 scaffold sequences, with an $\mathrm{N} 50$ of $2.76 \mathrm{Mb}$, and 78,313 contig sequences, with an N50 of $44.84 \mathrm{~Kb}$, for a total assembly size of $470.51 \mathrm{Mb}$ (Table 1). Completeness assessment was performed using Benchmarking Universal SingleCopy Orthologs (BUSCO) version 3.0.1 ${ }^{14}$ with Embryophyta odb9. The results showed that 1327 (92.20\%) of the expected 1440 conserved plant orthologs were detected as complete (Supplementary Table 1). To further evaluate the completeness of the assembled genome, we performed short read mapping using clean raw data with

Table 1 Statistics of genome assembly.

\begin{tabular}{|c|c|c|c|c|}
\hline & \multicolumn{2}{|l|}{ Contig } & \multicolumn{2}{|l|}{ Scaffold } \\
\hline & Size (bp) & Number & Size (bp) & Number \\
\hline $\mathrm{N} 90^{\mathrm{a}}$ & 5420 & 12,457 & 7033 & 3988 \\
\hline N80 & 13,875 & 7548 & 39,210 & 608 \\
\hline N70 & 23,325 & 5165 & 34,6109 & 131 \\
\hline N60 & 33,460 & 3619 & $1,307,770$ & 60 \\
\hline N50 & 44,841 & 2503 & $2,757,598$ & 35 \\
\hline Longest & 717,770 & - & $14,768,062$ & - \\
\hline Total size & $431,262,337$ & - & $470,508,511$ & - \\
\hline Total number ( $\geq 2 \mathrm{~kb}$ ) & - & 18,820 & - & 10,777 \\
\hline $\begin{array}{l}\text { Total number } \\
\text { ( } \geq 100 \mathrm{bp})\end{array}$ & - & 78,313 & - & 69,402 \\
\hline
\end{tabular}

${ }^{\mathrm{a}} \mathrm{N} x \mathrm{x}$ length is the maximum length $(L)$ such that $x x \%$ of all nucleotides lie within contigs (or scaffolds) with a size of at least $L$.
BWA-MEM software ${ }^{15}$. In total, 943,278,896 (99.12\%) reads could be mapped to the genome, and $88.13 \%$ of them were properly paired (Supplementary Table 2).

\section{Genome annotation}

A total of $68.15 \%$ of the assembled $A$. carambola genome was composed of repetitive elements (Supplementary Table 3). Among these repetitive sequences, LTRs were the most abundant, accounting for $61.64 \%$ of the genome. DNA class repeat elements represented $4.19 \%$ of the genome; LINE and SINE classes accounted for $0.28 \%$ and $0.016 \%$ of the assembled genome, respectively. For gene prediction, we combined homology-based and de novobased approaches and obtained a non-redundant set of 24,726 gene models with 4.11 exons per gene on average. The gene length was $3457 \mathrm{bp}$ on average, while the average exon and intron lengths were 215 and $827 \mathrm{bp}$, respectively. The gene model statistical data compared with seven other closely related species are shown in Supplementary Fig. 3. To evaluate the completeness of the gene models for $A$. carambola, we used BUSCO with Embryophyta odb9. A total of 1281 (88.9\%) complete orthologs were detected from the predicted star fruit gene sets (Supplementary Table 4).

Functions were assigned to 16,490 (66.69\%) genes. These protein-coding genes were then subjected to further exploration against the KEGG, NR, and COG protein sequence databases ${ }^{16}$, in addition to the SwissProt and TrEMBL databases ${ }^{17}$, and InterProScan ${ }^{18}$ was finally used to identify domains and motifs (Supplementary Table 5, Supplementary Fig. 4). Noncoding RNA genes in the assembled genome were also annotated. We predicted 759 tRNA, 1341 rRNA, 90 microRNA (miRNA), and 2039 small nuclear RNA (snRNA) genes in the assembled genome (Supplementary Table 6).

Since star fruit is an important cultivated plant, the identification of disease resistance genes was one of the focuses of our study. Nucleotide-binding site (NBS) genes play an important role in pathogen defense and the cell cycle. We identified a total of 80 non-redundant NBSencoding orthologous genes in the star fruit genome (Supplementary Table 7). Among these genes, TIR (encoding the toll interleukin receptor) motif was found to be significantly smaller than in other eudicot plants, except for cocoa. Unlike other plants, the leucine-rich repeat (LRR) motif was not the most or second most common motif in the NBS gene family in star fruit ${ }^{19}$.

\section{Genome evolution}

The characterization of the star fruit genome can provide necessary data for further analyzing the evolutionary history of Oxalidaceae. A $\gamma$ whole-genome triplication event affected over $75 \%$ of extant angiosperms and was associated with the early diversification of the core 


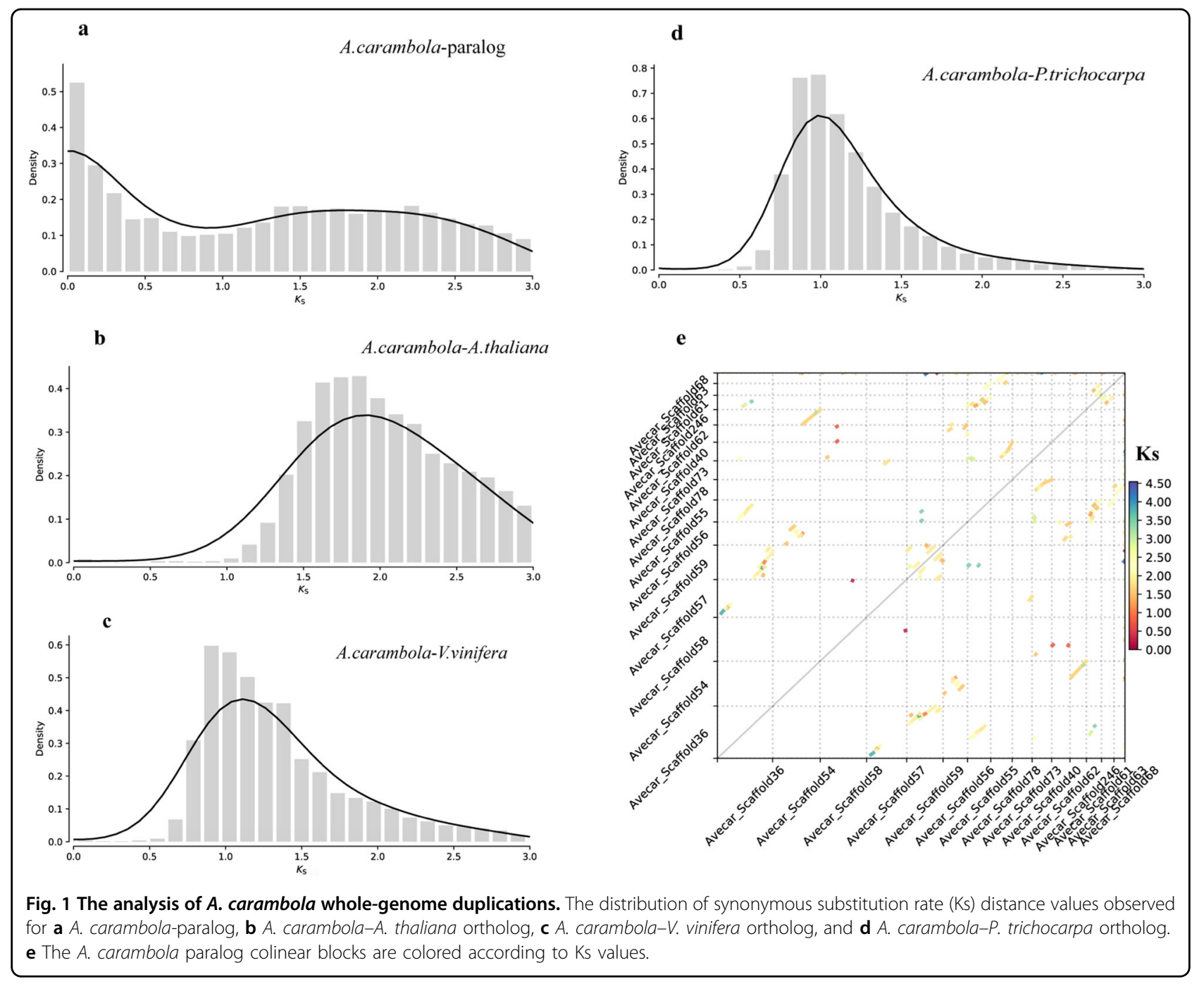

eudicots. To investigate evolutionary events at the genomic level in star fruit, we identified 1134 paralogous gene families on the basis of the 24,726 gene models. The synonymous substitution rates (Ks) in the duplicated genes $(K s=1.9)$ suggested that an ancient $\gamma$ event occurred in star fruit (Fig. 1a). Furthermore, we assessed the intergenomic collinearity among the Arabidopsis ${ }^{20}$, poplar $^{21}$, and grape ${ }^{22}$ genomes and identified relationships among star fruit orthologues. The mean Ks values from the one-to-one orthology analysis of star fruit in relation to Arabidopsis, poplar, and grape were 1.8, 1.0, and 1.2, respectively (Fig. 1b-d). The results confirmed the shared ancient whole-genome duplications (WGDs) event between the four species. Moreover, we generated whole-genome syntenic dotplots of star fruit based on the Ks value (Fig. 1e). Over $50 \%$ of the syntenic blocks shared a Ks rate between 1.0 and 2.0, and only $\sim 10 \%$ of the gene pairs exhibited a Ks below 1.0, which indicated that no recent WGDs have occurred in the star fruit genome.
Gene family analysis and phylogenetic tree

We performed $A$. carambola gene family analysis using OrthoMCL software ${ }^{23}$ with protein and nucleotide sequences from $A$. carambola and 10 other plant species (A. thaliana, C. sinensis, F. sylvatica, G. max, K. fedtschenkoi, $M$. domestica, $P$. granatum, $P$. trichocarpa, $T$. cacao, $V$. vinifera) based on an all-versus-all BLASTP alignment with an $E$-value cutoff of $1 \mathrm{e}-05$. The 24,726 predicted protein-coding genes in A. carambola were assigned to 9731 gene families consisting of 15,301 genes, while 9425 genes were not organized into groups and were unique to A. carambola (Supplementary Table 8, Fig. 2b). In total, 163 single-copy orthologs corresponding to the 11 species were extracted from the clusters and used to construct the phylogenetic tree. The constructed tree topology supported the APG IV ${ }^{24}$ system in which Oxalidales (A. carambola) and Malpighiales ( $P$. trichocarpa) are sister clades that belong to the same cluster (rosids). Based on the phylogenetic tree, A. carambola was 


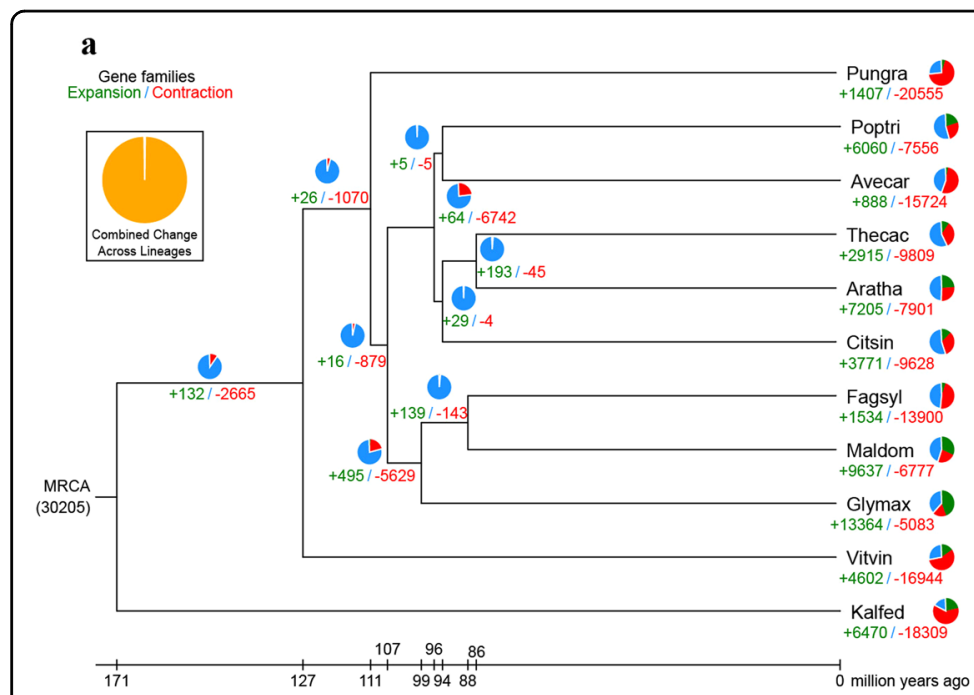

b

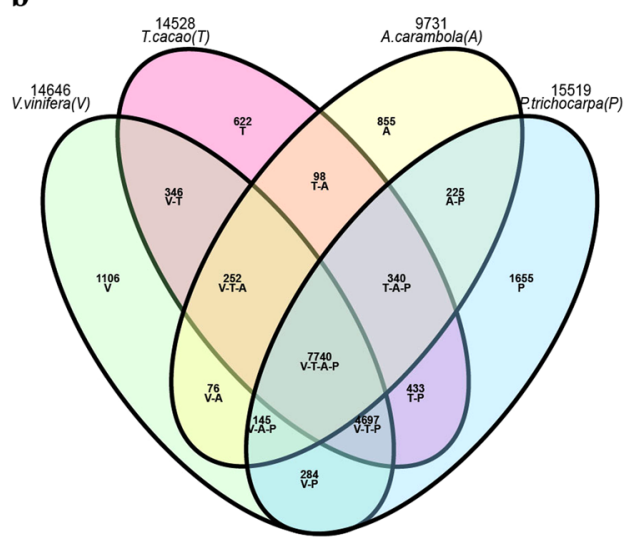

Fig. 2 Gene family analysis and phylogenetic tree construction. a Phylogenetic tree showing the sizes of expanded and contracted gene families. Pungra: P. granatum; Poptri: P. trichocarpa; Avecar: A. carambola; Thecac: T. cacao; Aratha: A. thaliana; Citsin: C. sinensis; Fagsyl: F. sylvatica; Maldom: M. domestica; Glymax: G. max; Vitvin: V. vinifera; Kalfed: K. fedtschenkoi. $\mathbf{b}$ Venn diagram of the number of shared gene families within A. carambola, V. vinifera, T. cacao, and P. trichocarpa.

estimated to have separated from P. trichocarpa, $V$. vinifera, and K. fedtschenkoi approximately 94.5, 110.2, and 126.3 Mya, respectively (Supplementary Fig. 5).

We also analyzed the expansion and contraction of the gene families between species using CAFÉ ${ }^{25}$. The results showed that 888 gene families were substantially expanded and that 15,724 gene families were contracted in $A$. carambola (Fig. 2a). In total, 2916 and 6057 genes identified in A. carambola came from expanded and contracted families, respectively, where contraction was approximately two times more common than expansion.

Gene ontology (GO) and KEGG functional enrichment analyses were subsequently performed for all expanded gene families. The KEGG pathway enrichment analysis results are shown in Table 2, and the GO-enrichment results are listed in Supplementary Table 9. In a previous study, several flavonoids were isolated from the fresh fruit of $A$. carambola, which are known to reduce harmful inflammation $^{26}$. In our study, the flavonoid biosynthesis pathway was found to be significantly enriched among the expanded families. Terpenoids are yet another important type of compound that has been isolated from star fruit ${ }^{27}$ and has been proven to exhibit anti-inflammatory activities. A. carambola likely synthesizes terpenoids via the diterpenoid biosynthesis pathway.

\section{Genes specifically involved in star fruit nutrition pathways}

Star fruit is an excellent source of various minerals and vitamins, especially natural antioxidants, such as Lascorbic acid (vitamin $C$ ) and riboflavin (vitamin $\left.\mathrm{B}_{2}\right)^{1,26}$. Through the ortholog searches in KEGG pathways, we identified enzymes that are potentially involved in the vitamin $\mathrm{C}$ and vitamin $\mathrm{B}_{2}$ biosynthesis pathways in $A$. carambola.

In a previous report, a major component of plant ascorbate was reported to be synthesized through the Lgalactose pathway ${ }^{28}$, in which GDP-D-mannose is converted to L-ascorbate through four successive intermediates, as summarized in Fig. 3a. Laing et al. ${ }^{29}$ reported the identification of L-galactose guanylyltransferaseencoding homologous genes from Arabidopsis and kiwifruit that encode enzymes that catalyze the conversion of GDP-L-galactose to L-galactose-1-P. In this study, five necessary enzymes (GalDH, GalLDH, GGalPP, GalPP, and GME) involved in the vitamin $C$ pathway were identified, suggesting the possibility of ascorbic acid synthesis in star fruit (Table 3). For L-galactose dehydrogenase (GalDH), we identified four paralogous genes in the star fruit genome. The copy number of GalDH genes in star fruit is close to that in tomato (5) and papaya (4) but approximately half that in other species (10 in poplar, 11 in orange, 8 in Arabidopsis, and 13 in grape, Supplementary Table 12). Further evolutionary analysis showed three clusters in the phylogenetic tree, and the most ancient cluster comprised all the grape genes. Among the other two sister clusters, one is ancient and comes from poplar, including four genes, and the other is closer to orange, including seven genes. The four genes in star fruit are divided into two clusters and were recently separated from their ancestors (Fig. 3b).

GalDH L-galactose dehydrogenase, GalLDH L-galactono-1,4-lactone dehydrogenase, GGalPP GDP-L- 
Table 2 Enriched KEGG pathways of unique genes of $A$. carambola showing expansion.

\begin{tabular}{|c|c|c|c|}
\hline Pathway ID & KEGG description & $\begin{array}{l}\text { Adjusted } P \text { - } \\
\text { value } \\
(\leq 0.05)\end{array}$ & $\begin{array}{l}\text { Number } \\
\text { of genes }\end{array}$ \\
\hline map03020 & RNA polymerase & 3.51E-09 & 30 \\
\hline map00904 & Diterpenoid biosynthesis & 1.00E-05 & 18 \\
\hline map00240 & Pyrimidine metabolism & $3.16 \mathrm{E}-05$ & 34 \\
\hline map01100 & Metabolic pathways & 7.17E-05 & 234 \\
\hline map00901 & Indole alkaloid biosynthesis & 7.17E-05 & 15 \\
\hline map00230 & Purine metabolism & 7.17E-05 & 36 \\
\hline map00565 & Ether lipid metabolism & 0.00014092 & 14 \\
\hline map01110 & $\begin{array}{l}\text { Biosynthesis of secondary } \\
\text { metabolites }\end{array}$ & 0.00038039 & 142 \\
\hline map02010 & $\mathrm{ABC}$ transporters & 0.00080624 & 20 \\
\hline map00902 & Monoterpenoid biosynthesis & 0.00116462 & 9 \\
\hline map00460 & Cyanoamino acid metabolism & 0.00270665 & 18 \\
\hline map00941 & Flavonoid biosynthesis & 0.03063434 & 17 \\
\hline map00190 & Oxidative phosphorylation & 0.0354555 & 19 \\
\hline map00940 & Phenylpropanoid biosynthesis & 0.0354555 & 32 \\
\hline map00062 & $\begin{array}{l}\text { Fatty acid elongation in } \\
\text { mitochondria }\end{array}$ & 0.0354555 & 7 \\
\hline map00563 & $\begin{array}{l}\text { Glycosylphosphatidylinositol } \\
\text { (GPI)-anchor biosynthesis }\end{array}$ & 0.03937265 & 8 \\
\hline map00040 & $\begin{array}{l}\text { Pentose and glucuronate } \\
\text { interconversions }\end{array}$ & 0.0426558 & 21 \\
\hline
\end{tabular}

galactose phosphorylase, GalPP L-galactose-1-phosphate phosphatase, GME GDP-D-mannose-3',5'-epimerase.

We also identified possible enzymes involved in the riboflavin (vitamin $\mathrm{B}_{2}$ ) biosynthesis pathway in star fruit (Fig. 4a, Table 3). Through catalysis by RIB3, RIB4, and RIB5, riboflavin can ultimately be produced from the Dribulose 5-phosphate compound. Furthermore, in the investigation of the possible biosynthesis pathway of the special product oxalate in star fruit, we identified three enzymes, citrate synthase (CS), isocitrate lyase (aceA), and aconitate hydratase (ACO), that can potentially catalyze the transformation of oxalacetic acid to glyoxylate within the glyoxylate and dicarboxylate metabolism pathway (Supplementary Table 10).

In the A. carambola gene family analysis, the KEGG pathway enrichment analysis of the expanded gene families revealed that 17 genes participate in the flavonoid synthesis pathway $(P$-value $=0.03$, Table 3$)$. Previous studies have proved that flavonoids can be isolated from A. carambola and other plants from the Oxalidaceae family ${ }^{1,9,26,30-33}$. Here, we identified 11 enzymes that could be potentially involved in the flavonoid biosynthesis pathway (Fig. 4b, Supplementary Table 11). The two enzymes in the pathway showing the highest copy numbers are shikimate O-hydroxycinnamoyltransferase (HCT) and chalcone synthase (CHS), with 23 and 21 copies, respectively. Among the end-point products, apigenin, cyanidin, epicatechin, and quercetin have been extracted from the leaves, fruits or bark of $A$. carambola in previous studies ${ }^{6,34-36}$.

\section{Discussion}

This study presents the first draft genome in the Oxalidaceae family. The sequenced species, A. carambola (star fruit), is widely cultivated and utilized as an edible fruit and serves as an important source of minerals and vitamins, in addition to presenting phytomedicinal properties. The assembled genome size was $470.51 \mathrm{Mb}$, with a scaffold N50 of $2.76 \mathrm{Mb}$. We cannot compare this genome size with those of other species in this family, but we found similar genome sizes in the closest order Malpighiales of $434.29 \mathrm{Mb}$ in Populus trichocarpa and $350.62 \mathrm{Mb}$ in Ricinus communis. However, the chromosome-level genome will be required to better understand the diploid character of this species.

In total, 24,726 gene models were identified from $A$. carambola. This gene number is relatively smaller than those from earlier reports for A. thaliana, P. trichocarpa, or $T$. cacao. The length distribution of the exons of the predicted star fruit proteins was consistent with those in other species, although the predicted intron and CDS lengths tended to be shorter than those in other species (Supplementary Fig. 3). The proportion of predicted genes containing an InterPro functional domain was $52.3 \%$, and the proportion that could be aligned with the NCBI nr database (66.4\%) was the highest among all databases. It is likely that $A$. carambola is the only species in the Oxalidaceae family whose genome has been assembled so far; there might be some evolutionarily unique genes in this family remaining to be annotated.

We subsequently performed gene family analysis together with the other 10 species and identified the significant expansion of 888 gene families containing 2916 unique genes in A. carambola. These genes were significantly enriched $(P$-value $\leq 0.05)$ within $28 \mathrm{GO}$ classes, including 18 biological process, 2 cellular component, and 8 molecular function categories (Supplementary Table 6). The biological process of DNA binding contained the most genes (60) within the expanded families. Within the significantly enriched biological processes, the defense response to fungus might be related to the antimicrobial property of star fruits identified in previous studies ${ }^{1}$. On the other hand, we found that oxidoreductase activity was enriched in the molecular function GO class, which could 
a

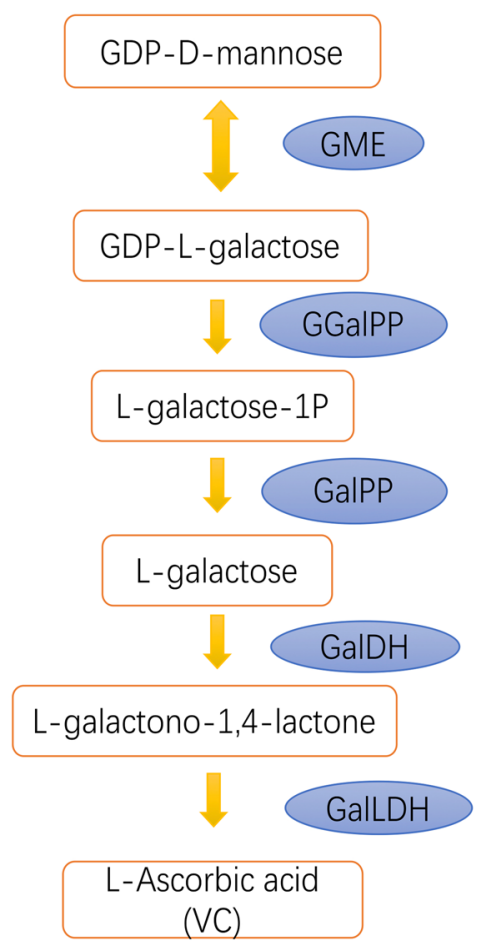

$\mathrm{b}$

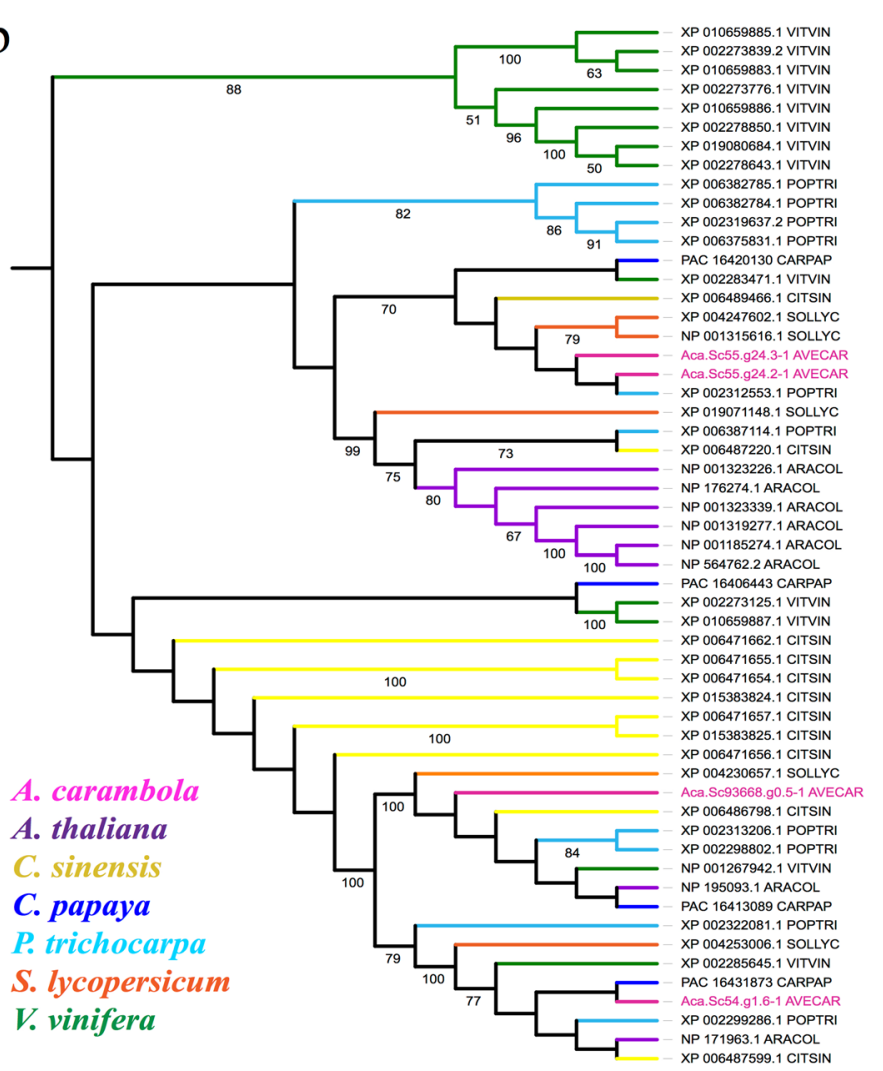

Fig. 3 Genes involved in vitamin C metabolism. a A proposed model for L-ascorbic acid biosynthesis pathways in star fruit. Genes identified as being involved in the pathways are shown in blue circles. $\mathbf{b}$ Phylogenetic analysis of the GalDH gene family in A. carambola (rose red), A. thaliana (purple), C. sinensis (yellow), C. papaya (blue), P. trichocarpa (sky blue), S. lycopersicum (orange), and V. vinifera (green).

be one of the potential reasons for the antioxidant activity of star fruits.

The genome evolution analysis indicated that star fruit only shared an ancient $\gamma$ event, and no recent WGD was observed. In a genome evolution study of poplar, which belongs to Malpighiales, the existence of a hexaploidization event and recent duplication were reported ${ }^{21}$. This result could partially explain why star fruit has half the number of gene sets compared to poplar.

Moreover, among the enriched KEGG pathways, we identified enzymes involved in nutritional metabolic pathways, including the vitamin $C$, vitamin $B_{2}$, oxalate, and flavonoid pathways. Although potential functional enzymes have been identified from the genome, these functional pathways should be verified by experimental studies in the future.

In summary, it can be expected that this draft genome will facilitate the elucidation of the development of specific important traits in star fruit plants, such as the biosynthesis pathways of pharmacologically active metabolites, and will contribute to the improvement of breeding strategies for star fruit plants.

\section{Materials and methods}

Plant materials and sequencing

Leaf samples of $A$. carambola were collected from the Ruili Botanical Garden, Yunnan, China. Genomic DNA was extracted by using the cetyl-triethylammonium bromide (CTAB) method $^{37}$. 10X Genome sequencing was performed to obtain high-quality reads. High-molecularweight (HMW) DNA was loaded onto a Chromium Controller chip with 10X Chromium reagents and gel beads, and the rest of the procedures were carried according to the manufacturer's protocol ${ }^{38}$. Then, the BGISEQ-500 platform was used to produce $2 \times 150 \mathrm{bp}$ paired-end sequences, generating a total of $206.28 \mathrm{~Gb}$ of raw data. The raw reads were filtered using SOAPfilter v2.2 with the following parameters: "-q $33-\mathrm{i} 600-\mathrm{p}-\mathrm{l}-\mathrm{f}-\mathrm{z}$ -g 1 -M 2 -Q 20". After filtering low-quality reads, $\sim 114.42 \mathrm{~Gb}$ of clean data (high-quality reads >Q35) remained for the next step.

\section{Estimation of $A$. carambola genome size}

All $114.42 \mathrm{~Gb}$ clean reads obtained from the BGISEQ500 platform were subjected to 17 kmer frequency 
Table 3 List of genes involved in the vitamin $C$ and vitamin $B_{2}$ pathways.

\begin{tabular}{|c|c|c|c|c|c|}
\hline Pathway & Enzyme & Description & Copy numbers & Gene ID & Protein (AA) \\
\hline \multirow[t]{16}{*}{ Vitamin C pathway } & \multirow[t]{4}{*}{ GalDH } & \multirow[t]{4}{*}{ L-galactose dehydrogenase } & \multirow[t]{4}{*}{4} & Aca.sc093668.g0.5 & 332 \\
\hline & & & & Aca.sc000054.g1.6 & 334 \\
\hline & & & & Aca.sc000055.g24.2 & 351 \\
\hline & & & & Aca.sc000055.g24.3 & 594 \\
\hline & \multirow[t]{5}{*}{ GalLDH } & \multirow[t]{5}{*}{ L-galactono-1,4-lactone dehydrogenase } & \multirow[t]{5}{*}{5} & Aca.sc000059.g19.35 & 601 \\
\hline & & & & Aca.sc000229.g0.29 & 603 \\
\hline & & & & Aca.sc000078.g43.5 & 598 \\
\hline & & & & Aca.sc100684.g0.3 & 99 \\
\hline & & & & Aca.sc102574.g0.3 & 99 \\
\hline & \multirow[t]{3}{*}{ GGalPP } & \multirow[t]{3}{*}{ GDP-L-galactose phosphorylase } & \multirow[t]{3}{*}{3} & Aca.sc150475.g0.5 & 275 \\
\hline & & & & Aca.sc150564.g0.4 & 288 \\
\hline & & & & Aca.sc098705.g0.4 & 410 \\
\hline & \multirow[t]{2}{*}{ GalPP } & \multirow[t]{2}{*}{ L-galactose-1-phosphate phosphatase } & \multirow[t]{2}{*}{2} & Aca.sc006151.g1 & 181 \\
\hline & & & & Aca.sc000246.g13.57 & 360 \\
\hline & \multirow[t]{2}{*}{ GME } & \multirow[t]{2}{*}{ GDP-D-mannose-3',5'-epimerase } & \multirow[t]{2}{*}{2} & Aca.sc096116.g0.5 & 383 \\
\hline & & & & Aca.sc000061.g13.42 & 383 \\
\hline \multirow[t]{7}{*}{ Vitamin $B_{2}$ pathway } & \multirow[t]{3}{*}{ RIB3 } & \multirow[t]{3}{*}{ 3,4-dihydroxy 2-butanone 4-phosphate synthase } & \multirow[t]{3}{*}{3} & Aca.sc000063.g38.15 & 545 \\
\hline & & & & Aca.sc000056.g65.27 & 572 \\
\hline & & & & Aca.sc000071.g11.34 & 590 \\
\hline & RIB4 & 6,7-dimethyl-8-ribityllumazine synthase & 1 & Aca.sc023103.g76.2 & 185 \\
\hline & RIB5 & riboflavin synthase & 1 & Aca.sc000036.g2.16 & 343 \\
\hline & RFK & riboflavin 5'-phosphotransferase & 1 & Aca.sc000058.g77.42 & 539 \\
\hline & FLAD1 & FMN adenylyltransferase & 1 & Aca.sc000058.g25.58 & 520 \\
\hline
\end{tabular}

distribution analysis with Jellyfish ${ }^{39}$ using the parameters "-k 17 -t 24". The frequency graph was drawn, and the $A$. carambola genome size was calculated using the formula: genome size $=$ k-mer_Number/Peak_Depth.

\section{De novo genome assembly}

The linked read data were assembled using Supernova v2.1.1 software ${ }^{13}$ using the "--localcores $=24$--localmem $=350$--max reads 280000000 " parameter. To fill the scaffold gaps, GapCloser version $1.12^{40}$ was used with the parameters "-t 12 -1 155". Finally, the total assembled length of the $A$. carambola genome was $470.51 \mathrm{Mb}$, with a scaffold N50 of $275.76 \mathrm{~Kb}$ and a contig N50 of $44.84 \mathrm{~Kb}$.

\section{Repeat annotation}

For transposable element annotation, RepeatMasker $\mathrm{v} 3.3 .0^{41}$ and RepeatProteinMasker v3.3.0 ${ }^{41}$ were applied against Repbase v16.10 ${ }^{42}$ to identify known repeats in the A. carambola genome. Tandem repeats were identified using Tandem Repeats Finder v4.07b ${ }^{43}$. De novo repeat identification was conducted using the RepeatModeler v1.0.5 ${ }^{44}$ and LTR_FINDER v1.05 $5^{45}$ programs, followed by RepeatMasker v3.3.0 ${ }^{41}$ to obtain the final results.

\section{Gene prediction}

Prior to the gene prediction analysis, we masked the repetitive regions of the genome. MAKER-P v2.31 ${ }^{46}$ was utilized to predict protein-coding genes based on homology and de novo prediction evidence. For homology-based prediction, the protein sequences of Theobroma cacao, Prunus persica, Prunus mume, Prunus avium, Populus trichocarpa, Populus euphratica, and Arabidopsis thaliana were aligned against the $A$. carambola genome using BLAT ${ }^{47}$. Then, the gene structure was predicted using GeneWise ${ }^{48}$. To optimize different $a b$ initio gene predictors, we constructed a series of training sets for de novo prediction data. Complete gene models according to homology evidence were picked for training with the Augustus tool ${ }^{49}$. Genemark-ES v4.21 ${ }^{50}$ was selftrained using the default criteria. The first round of 


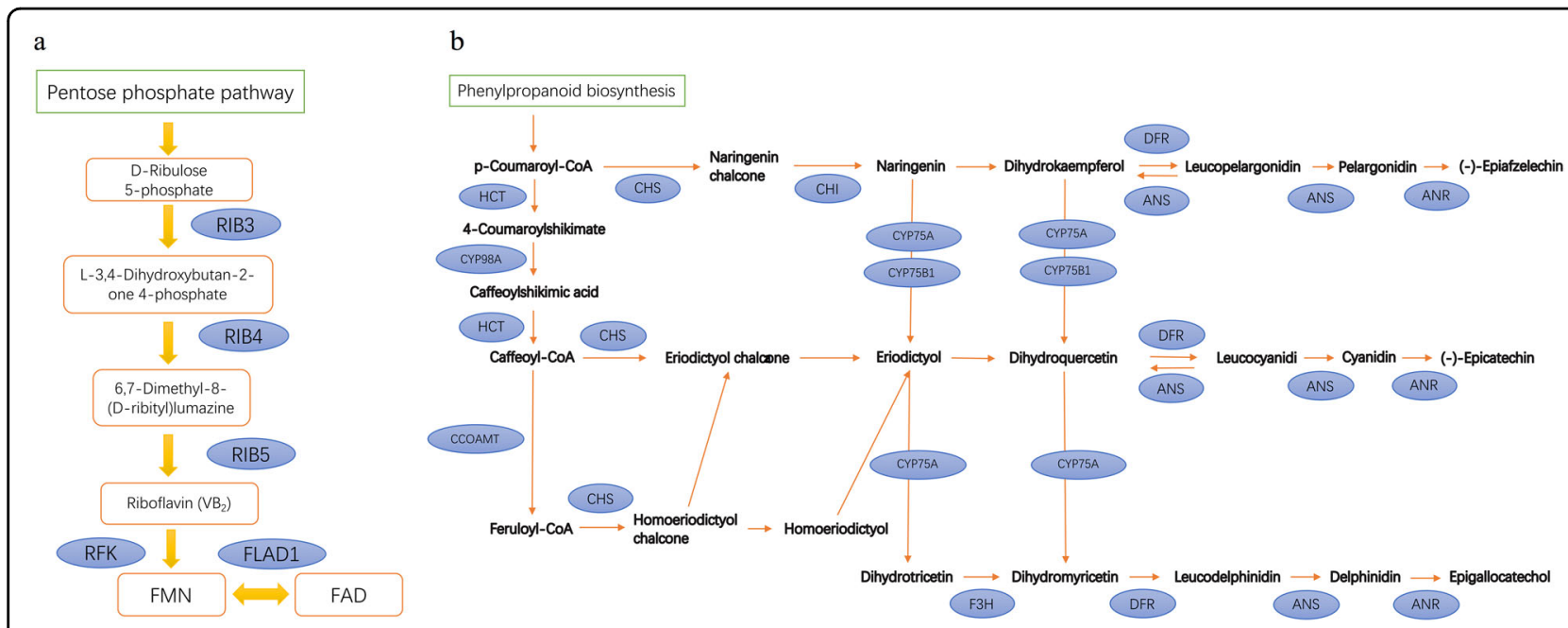

Fig. 4 Identification of genes involved in the (a) riboflavin and (b) flavonoid biosynthesis pathways. Genes identified as being involved in the two pathways are shown in blue circles. FLAD1 FMN adenylyltransferase, RIB3 3,4-dihydroxy 2-butanone 4-phosphate synthase, RIB4 6,7-dimethyl-8ribityllumazine synthase, RIB5 riboflavin synthase, RFK riboflavin 5'-phosphotransferase, ANR anthocyanidin reductase, ANS leucoanthocyanidin dioxygenase, CCOAMT caffeoyl-COA O-methyltransferase, CHI chalcone isomerase, CHS chalcone synthase, CYP75A flavonoid 3',5'-hydroxylase, CYP75B1 flavonoid 3'-monooxygenase, CYP98A coumaroylquinate 3'-monooxygenase, DFR flavanone 4-reductase, F3H naringenin 3-dioxygenase, HCT shikimate O-hydroxycinoyltransferase.

MAKER-P analysis was also run using the default parameters on the basis of the above evidence, with the exception of "protein2genome", which was set to "1", yielding only protein-supported gene models. $\mathrm{SNAP}^{51}$ was then trained with these gene models. The default parameters were used to run the second and final rounds of MAKER-P, generating the final gene models.

\section{Functional annotation}

The predicted gene models were functionally annotated by aligning their protein sequences against the Kyoto Encyclopedia of Genes and Genomes (KEGG) ${ }^{52}$, Clusters of Orthologous Groups (COG) ${ }^{16}$, SwissProt ${ }^{17}$, TrEMBL, and National Center for Biotechnology Information (NCBI) non-redundant (NR) protein databases with BLASTP $(E$-value $\leq 1 \mathrm{e}-05)$. Protein motifs and domains were identified by comparing the sequences against various domain databases, including the PFAM, PRINTS, PANTHER, ProDom, PROSITE, and SMART databases, using InterProScan v5.21 $1^{18}$. For ncRNA annotation, tRNA genes were identified with tRNAscan-SE v1.23 ${ }^{53}$. For the identification of rRNA genes, we aligned the assembled data against the rRNA sequences of $A$. thaliana using BLASTN ( $E$-value $\leq 1 \mathrm{e}-05)$. miRNAs and snRNAs were predicted by using INFERNAL ${ }^{54}$ software against the Rfam database ${ }^{55}$.

To classify the NBS domains in the star fruit protein sequences, we used hidden Markov models (HMM) to search for the Pfam NBS (NB-ARC) family PF00931 with an $E$-value cutoff of 1.0. To detect TIR domains, the amino acid sequences were also screened by using the HMM model Pfam TIR PF01582 $(E$-value $\leq 1.0)$. Moreover, to identify LRR motifs, we performed an HMM search for Pfam LRR models $(E$-value $\leq 1.0)$ against star fruit NBS-encoding amino acid sequences.

To compare the orthologous genes in the vitamin $C$, vitamin $\mathrm{B}_{2}$, flavonoid and oxalate pathways between other plant species ( $P$. trichocarpa, C. sinensis, A. thaliana, S. lycopersicum, C. papaya, and V. vinifera), we also annotated the protein sequences by aligning them against the KEGG database ${ }^{52}$ with BLASTP $(E-$ value $\leq 1 \mathrm{e}-05)$ and then performed filtering according to Pfam domains annotated using InterProScan $\mathrm{v} 5.21^{18}$.

\section{Genome evolution}

The genome sequences used for orthology analysis were downloaded from NCBI (https://www.ncbi.nlm. nih.gov) for $A$. thaliana (GCA_000001735.2), V. vinifera (GCA_000003745.2), and P. trichocarpa (GCA 000002775.3). Next, we used wgd software ${ }^{56}$ to perform the Ks distribution analysis. The analyses of paralogous gene families and one-to-one orthologs were performed using the "wgd mcl" command. Then, the Ks distribution for a set of paralogous families or one-to-one orthologs was constructed using "wgd ksd". Next, we applied the "wgd kde" command for the fitting of kernel density estimates (KDEs). Finally, the colinear blocks of the A. carambola paralog were identified by I-ADHoRe ${ }^{57}$ and colored according to their median Ks value. 


\section{Gene family construction and phylogenetic analysis}

For gene family analysis, OrthoMCL ${ }^{23}$ software was utilized to construct the orthologous gene families of all the protein-coding genes of $A$. carambola and other 10 sequenced plant species (A. thaliana, C. sinensis, F. sylvatica, G. max, K. fedtschenkoi, $M$. domestica, P. granatum, P. trichocarpa, T. cacao, $V$. vinifera). Before the application of OrthoMCL, BLASTP was used to find similar matches from different species with an $E$-value cutoff of 1e-05. The composition of the OrthoMCL clusters was used to calculate the total number of gene families.

Orthogroups that were present in a single copy in all species analyzed were selected and aligned using MAFFT v7.310 $0^{58}$. Each gene tree was constructed by using RAxML v8.2. $4^{59}$ with the GTRGAMMA model. To construct the species phylogenetic tree, a coalescent-based method in ASTRAL v4.10.4 ${ }^{60}$ with 100 replicates of multilocus bootstrapping ${ }^{61}$ was used.

The divergence time between $A$. carambola and other species was estimated using MCMCTREE ${ }^{59}$ with the default parameters. The expansion and contraction of gene family numbers were predicted using $\mathrm{CAFE}^{25}$ by employing the phylogenetic tree and gene family statistics.

To further perform the phylogenetic analysis of the key enzyme GalDH in the vitamin C pathway, we annotated orthologous genes from six other plant species $(A$. thaliana, C. papaya, C. sinensis, $P$. trichocarp, $V$. vinifera, $S$. lycopersicum) using BLASTP with an $E$-value cutoff of $1 \mathrm{e}-05$ to align coding sequences against the KEGG database. In total, 55 orthologous genes were used to generate a phylogenetic tree via the maximum likelihood (ML) method in RAxML $v 8.2 .4^{59}$, and 20 runs were included to identify an optimal tree using the GTRGAMMA substitution model and 100 nonparametric bootstrap replicates.

\section{Acknowledgements}

This work was supported by funding from the National Key R\&D Program of China (No. 2019YFC1711000), the Shenzhen Municipal Government of China (grants JCYJ20170817145512476 and JCYJ20160510141910129), the Guangdong Provincial Key Laboratory of Genome Read and Write (grant 2017B030301011), and the NMPA Key Laboratory for the Rapid Testing Technology of Drugs.

\section{Author details}

'State Key Laboratory of Agricultural Genomics, China National GeneBank, BGIShenzhen, 518120 Shenzhen, China. ${ }^{2}$ BGI-Yunnan, BGI-Shenzhen, 650106 Kunming, China. ${ }^{3}$ Forestry Bureau of Ruili, Yunnan Dehong, 678600 Ruili, China. ${ }^{4}$ Chinese Academy of Forestry, Beijing, China. ${ }^{5}$ Guangdong Provincial Key Laboratory of Genome Read and Write, 518120 Shenzhen, China. ${ }^{6} \mathrm{BGI}-$ Fuyang, BGI-Shenzhen, 236009 Fuyang, China. ${ }^{7}$ Department of Biology, University of Copenhagen, Copenhagen, Denmark

\section{Author contributions}

R.-c.M., J.L., J.-m.Z., T.Y., Y.-X.Z. and W.-X.M. collected the samples; W.-X.M. and S. K.S. conceived and conducted the experiments; Y.-n.F. and T.Y. analyzed the results; Y.-n.F. and S.K.S. wrote the manuscript.

\section{Data availability}

The datasets generated and analyzed during the current study are available in the CNGB Nucleotide Sequence Archive (CNSA: https://db.cngb.org/cnsa). The raw sequencing data are under ID CNR0066625, and assembly data are under ID CNA0002506. All other data generated or analyzed during this study are included in this published article and its supplementary information files.

\section{Conflict of interest}

The authors declare that they have no conflicts of interest.

Supplementary Information accompanies this paper at (https://doi.org/ 10.1038/s41438-020-0306-4).

Received: 6 November 2019 Revised: 3 January 2020 Accepted: 14 February 2020

Published online: 01 June 2020

\section{References}

1. Muthu, N., Lee, S. Y., Phua, K. K. \& Bhore, S. J. Nutritional, medicinal and toxicological attributes of star-fruits (Averrhoa carambola L.): a review. Bioinformation 12, 420-424 (2016).

2. Khoo, $\mathrm{H}$. et al. A review on underutilized tropical fruits in Malaysia. Guangxi Agric. Sci. 41, 698-702 (2010).

3. Ray, P. K. Breeding Tropical and Subtropical Fruits, XVI. 338 (Springer-Verlag, Berlin, 2002).

4. Wu, S.-C., Wu, S.-H. \& Chau, C.-F. Improvement of the hypocholesterolemic activities of two common fruit fibers by micronization processing. J. Agric. Food Chem. 57, 5610-5614 (2009).

5. Cabrini, D. A. et al. Analysis of the potential topical anti-inflammatory activity of Averrhoa carambola L. in mice. Evid. Based Complement. Altern. Med. 2011, 908059-908059 (2011).

6. Shui, G. \& Leong, L. P. Analysis of polyphenolic antioxidants in star fruit using liquid chromatography and mass spectrometry. J. Chromatogr. A 1022, 67-75 (2004).

7. Annegowda, H. V., Bhat, R., Min-Tze, L., Karim, A. A. \& Mansor, S. M. Influence of sonication treatments and extraction solvents on the phenolics and antioxidants in star fruits. J. Food Sci. Technol. 49, 510-514 (2012).

8. Cazarolli, L. H. et al. Anti-hyperglycemic action of apigenin-6-C$\beta$-fucopyranoside from Averrhoa carambola. Fitoterapia 83, 1176-1183 (2012).

9. Suluvoy, J. K., Sakthivel, K. M., Guruvayoorappan, C. \& Berlin Grace, V. M. Protective effect of Averrhoa bilimbi L. fruit extract on ulcerative colitis in wistar rats via regulation of inflammatory mediators and cytokines. Biomed. Pharmacother. 91, 1113-1121 (2017).

10. Wei, X. et al. Protective effects of 2-dodecyl-6-methoxycyclohexa-2,5-diene-1,4dione isolated from Averrhoa carambola L. (Oxalidaceae) roots on neuron apoptosis and memory deficits in Alzheimer's disease. Cell. Physiol. Biochem. 49, 1105-1114 (2018).

11. Cheng, S. et al. 10KP: a phylodiverse genome sequencing plan. Gigascience 7, giy013 (2018)

12. Liu, H. et al. Molecular digitization of a botanical garden: high-depth whole genome sequencing of 689 vascular plant species from the Ruili Botanical Garden. GigaScience 8, 1-9 (2019).

13. Weisenfeld, N. I., Kumar, V., Shah, P., Church, D. M. \& Jaffe, D. B. Direct determination of diploid genome sequences. Genome Res. 27, 757-767 (2017).

14. Waterhouse, R. M. et al. BUSCO applications from quality assessments to gene prediction and phylogenomics. Mol. Biol. Evol. 35, 543-548 (2017).

15. Vasimuddin, M. et al. Efficient Architecture-Aware Acceleration of BWA-MEM for Multicore Systems. IEEE International Parallel and Distributed Processing Symposium (IPDPS), Rio de Janeiro, Brazil, 314-324 (2019).

16. Tatusov, R. L., Galperin, M. Y., Natale, D. A. \& Koonin, E. V. The COG database: a tool for genome-scale analysis of protein functions and evolution. Nucleic Acids Res. 28, 33-36 (2000).

17. Bairoch, A. \& Apweiler, R. The SWISS-PROT protein sequence database and its supplement TrEMBL in 2000. Nucleic Acids Res. 28, 45-48 (2000).

18. Quevillon, E. et al. InterProScan: protein domains identifier. Nucleic Acids Res. 33. W116-W120 (2005).

19. Argout, X. et al. The genome of Theobroma cacao. Nat. Genet. 43, 101 (2011)

20. Swarbreck, D. et al. The Arabidopsis Information Resource (TAIR): gene structure and function annotation. Nucleic Acids Res. 36, D1009-D1014 (2007). 
21. Tuskan, G. A. et al. The genome of black cottonwood, Populus trichocarpa (Torr. \& Gray). Science 313, 1596-1604 (2006).

22. Jaillon, $\mathrm{O}$. et al. The grapevine genome sequence suggests ancestral hexaploidization in major angiosperm phyla. Nature 449, 463 (2007).

23. Li, L., Stoeckert, C. J. \& Roos, D. S. OrthoMCL: identification of ortholog groups for eukaryotic genomes. Genome Res. 13, 2178-2189 (2003).

24. Chase, M. W. et al. An update of the Angiosperm Phylogeny Group classification for the orders and families of flowering plants: APG IV. Bot. J. Linn. Soc. 181, 1-20 (2016).

25. De Bie, T., Cristianini, N., Demuth, J. P. \& Hahn, M. W. CAFE: a computational tool for the study of gene family evolution. Bioinformatics 22, 1269-1271 (2006).

26. Jia, X., Xie, H., Jiang, Y. \& Wei, X. Flavonoids isolated from the fresh sweet fruit of Averrhoa carambola, commonly known as star fruit. Phytochemistry 153 156-162 (2018).

27. Moresco, H. H., Queiroz, G. S., Pizzolatti, M. G. \& Brighente, I. M. Chemical constituents and evaluation of the toxic and antioxidant activities of Averrhoa carambola leaves. Braz. J. Pharmacogn. 22, 319-324 (2012).

28. Wheeler, G. L., Jones, M. A. \& Smirnoff, N. The biosynthetic pathway of vitamin C in higher plants. Nature 393, 365 (1998).

29. Laing, W. A., Wright, M. A., Cooney, J. \& Bulley, S. M. The missing step of the Lgalactose pathway of ascorbate biosynthesis in plants, an L-galactose guanyltransferase, increases leaf ascorbate content. Proc. Natl Acad. Sci. USA 104 9534-9539 (2007).

30. Kurup, S. B. \& Mini, S. Averrhoa bilimbi fruits attenuate hyperglycemia-mediated oxidative stress in streptozotocin-induced diabetic rats. J. Food Drug Anal. 25, 360-368 (2017).

31. Liu, Y., Zhang, X. \& Tian, X. Extraction and purification of flavonoids in Carambola. J. Shenyang Agric. Univ. 40, 491-493 (2009).

32. Yang, D., Xie, H., Jia, X. \& Wei, X. Flavonoid C-glycosides from star fruit and their antioxidant activity. J. Funct. Foods 16, 204-210 (2015).

33. Jia, X., Xie, H., Jiang, Y. \& Wei, X. Phytochemistry flavonoids isolated from the fresh sweet fruit of Averrhoa carambola, commonly known as star fruit. Phytochem. 153, 156-162 (2018).

34. Moresco, H. H., Queiroz, G. S., Pizzolatti, M. G. \& Brighente, I. Chemical constituents and evaluation of the toxic and antioxidant activities of Averrhoa carambola leaves. Rev. Bras. Farmacogn. 22, 319-324 (2012).

35. Tiwari, K., Masood, M. \& Minocha, P. Chemical constituents of Gmelina phillipinensis, Adenocalymna nitida, Allamanda cathartica, Averrhoa carambola and Maba buxifolia. J. Indian Chem. Soc. (1979).

36. Gunasegaran, R. Flavonoids and anthocyanins of three Oxalidaceae. Fitoterapia 63, 89-90 (1992).

37. Sahu, S. K., Thangaraj, M. \& Kathiresan, K. DNA extraction protocol for plants with high levels of secondary metabolites and polysaccharides without using liquid nitrogen and phenol. ISRN Mol. Biol. 2012, 1-6 (2012).

38. 10X Genomics. https://www.10xgenomics.com (2017).

39. Marçais, G. \& Kingsford, C. A fast, lock-free approach for efficient parallel counting of occurrences of k-mers. Bioinformatics 27, 764-770 (2011).

40. Luo, R. et al. SOAPdenovo2: an empirically improved memory-efficient shortread de novo assembler. Gigascience 1, 18 (2012).
41. Tarailo-Graovac, M. \& Chen, N. Using RepeatMasker to identify repetitive elements in genomic sequences. Curr. Protoc. Bioinform. 25, 4.10.11-14.10.14 (2009).

42. Jurka, J. et al. Repbase Update, a database of eukaryotic repetitive elements. Cytogenet. Genome Res. 110, 462-467 (2005).

43. Benson, G. Tandem repeats finder: a program to analyze DNA sequences. Nucleic Acids Res. 27, 573-580 (1999).

44. Price, A. L., Jones, N. C. \& Pevzner, P. A. De novo identification of repeat families in large genomes. Bioinformatics 21, i351-i358 (2005).

45. Xu, Z. \& Wang, H. LTR_FINDER: an efficient tool for the prediction of full-length LTR retrotransposons. Nucleic Acids Res. 35, W265-W268 (2007).

46. Campbell, M. S., Holt, C., Moore, B. \& Yandell, M. Genome annotation and curation using MAKER and MAKER-P. Curr. Protoc. Bioinform. 48, 4.11.11-14.11.39 (2014).

47. Kent, W. J. BLAT-the BLAST-like alignment tool. Genome Res. 12, 656-664 (2002).

48. Birney, E., Clamp, M. \& Durbin, R. GeneWise and genomewise. Genome Res. 14, 988-995 (2004).

49. Stanke, M., Schöffmann, O., Morgenstern, B. \& Waack, S. Gene prediction in eukaryotes with a generalized hidden Markov model that uses hints from external sources. BMC Bioinform. 7, 62 (2006).

50. Lomsadze, A., Ter-Hovhannisyan, V., Chernoff, Y. O. \& Borodovsky, M. Gene identification in novel eukaryotic genomes by self-training algorithm. Nucleic Acids Res. 33, 6494-6506 (2005).

51. Korf, I. Gene finding in novel genomes. BMC Bioinform. 5, 59 (2004).

52. Kanehisa, M. \& Goto, S. KEGG: kyoto encyclopedia of genes and genomes. Nucleic Acids Res. 28, 27-30 (2000).

53. Lowe, T. M. \& Eddy, S. R. tRNAscan-SE: a program for improved detection of transfer RNA genes in genomic sequence. Nucleic Acids Res. 25, 955-964 (1997).

54. Nawrocki, E. P., Kolbe, D. L. \& Eddy, S. R. Infernal 1.0: inference of RNA alignments. Bioinformatics 25, 1335-1337 (2009).

55. Nawrocki, E. P. et al. Rfam 12.0: updates to the RNA families database. Nucleic Acids Res. 43, D130-D137 (2014).

56. Zwaenepoel, A. \& Van de Peer, Y. wgd_-simple command line tools for the analysis of ancient whole-genome duplications. Bioinformatics 35, 2153-2155 (2018).

57. Proost, S. et al. i-ADHoRe 3.0-fast and sensitive detection of genomic homology in extremely large data sets. Nucleic Acids Res. 40, e11 (2011).

58. Katoh, K. \& Standley, D. M. MAFFT multiple sequence alignment software version 7: improvements in performance and usability. Mol. Biol. Evol. 30 772-780 (2013).

59. Stamatakis, A. RAXML version 8: a tool for phylogenetic analysis and postanalysis of large phylogenies. Bioinformatics 30, 1312-1313 (2014).

60. Mirarab, S. et al. ASTRAL: genome-scale coalescent-based species tree estimation. Bioinformatics 30, i541-i548 (2014).

61. Seo, T.-K. Calculating bootstrap probabilities of phylogeny using multilocus sequence data. Mol. Biol. Evol. 25, 960-971 (2008). 